\title{
A 3D MODEL BASED INDOOR NAVIGATION SYSTEM FOR HUBEI PROVINCIAL MUSEUM
}

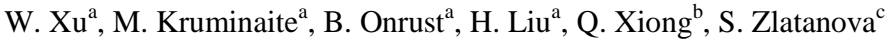

${ }^{a}$ Delft University of Technology, Faculty of Architecture and the Built Environment, Delft, The Netherlands, (W.Xu, M.Kruminaite, B.Onrust, H.Liu-2)@student.tudelft.nl

${ }^{\mathrm{b}}$ State Key Laboratory of Information Engineering in Survey, Mapping and Remote Sensing, Wuhan University,

Wuhan, China, whuxiong@whu.edu.cn

${ }^{c}$ GIS Technology, OTB - Research for the Built Environment, Faculty of Architecture and the Built Environment, Delft University of Technology, Jaffalaan 9, 2628 BX Delft, the Netherlands, +31 (0)15 278 2714, S.Zlatanova@tudelft.nl

KEY WORDS: Three-dimensional, Navigation, Mobile, Application, Database.

\begin{abstract}
:
3D models are more powerful than 2D maps for indoor navigation in a complicate space like Hubei Provincial Museum because they can provide accurate descriptions of locations of indoor objects (e.g., doors, windows, tables) and context information of these objects. In addition, the 3D model is the preferred navigation environment by the user according to the survey. Therefore a 3D model based indoor navigation system is developed for Hubei Provincial Museum to guide the visitors of museum. The system consists of three layers: application, web service and navigation, which is built to support localization, navigation and visualization functions of the system. There are three main strengths of this system: it stores all data needed in one database and processes most calculations on the webserver which make the mobile client very lightweight, the network used for navigation is extracted semi-automatically and renewable, the graphic user interface (GUI), which is based on a game engine, has high performance of visualizing 3D model on a mobile display.
\end{abstract}

\section{INTRODUCTION}

Built environment is an important factor in people's daily life as they spend most of their time indoors. It is extremely important for people to find their destination in large complex buildings, such as malls, airports and museums. So far most indoor navigation systems such as Google map (Google, n.d.), Wifarer (Wifarer Indoor Positioning, n.d.) and BuildNGO (Android Apps on Google Play, n.d.) use 2D maps to represent the environment. However, these maps do not provide adequate information about object features, such as 3D shape, colours and textures. Thus a 3D model has to be used, to overcome the limitations of 2D representation. A 3D model provides users with more realistic spatial information and offers more possibilities to represent vertical information such as height of windows and doors, stairs, irregular shapes (like non-vertical walls) or areas that are affected by moving obstacles such as smoke.

As mentioned in previous studies (Le et al., 2009; Afyouni et al., 2012; Fallah, et al., 2013), to be able to build such an application several components are needed: positioning method, a digital model, algorithms for path finding and a guidance mechanism (Becker et al., 2009). A digital model of building is one of the major components to ensure correct localization and suitable path finding to target location (room, part of a room or particular object).

The aim of this research was to explore the usability of a 3D model for indoor localisation and navigation by creating a mobile navigation application for Hubei Provincial Museum in Wuhan, China. A successful implementation of the proposed system would provide visitors of Hubei Provincial Museum with their current locations displayed in a 3D model of the museum on their mobile device and the shortest route when searching for an object of interest (e.g., exhibit, exit or restroom).

This paper is organised as follows. Section 2 elaborates on the need of 3D model for indoor navigation from both technique and user views. Section 3 provides the requirements for the $3 \mathrm{D}$ model and guidelines for its implementation. In section 4 the detailed implementation of the system is introduced with descriptions of database, system architecture and graphic user interface. Finally, the conclusions and recommendations are provided in section 5 .

\section{NEED OF 3D MODEL}

Indoor navigation differs from outdoor navigation substantially as the indoor environment is far more complex. The orientation in the building is complicated by the existence of multiple levels, relatively smaller spaces and the more difficult overview of the entire indoor environment. Moreover, people have the option to move freely within rooms and corridors in contrast to streets. Additionally indoors spaces have many obstacles such as furniture, columns and podiums which can be avoided in various ways. A 3D model of the building can represent the environment in more details and is expected that will help better in orientation and guidance (Lee, 2004).

3D model is considered to be a preferable representation of navigation environment of Hubei Provincial Museum due to the following technical reasons (Figure 1). Firstly, the museum has multiple floors with a number of theme exhibition rooms, thus the 3D model provides visitors with better understanding of the vertical paths linking different floors. Additionally, 3D model can provide visitors with information about specific exhibits and guide visitors to various exhibits inside the show room. Lastly, 3D model allows more semantic annotations to be presented, it 
makes possible to provide additional information on the items on the exhibit.
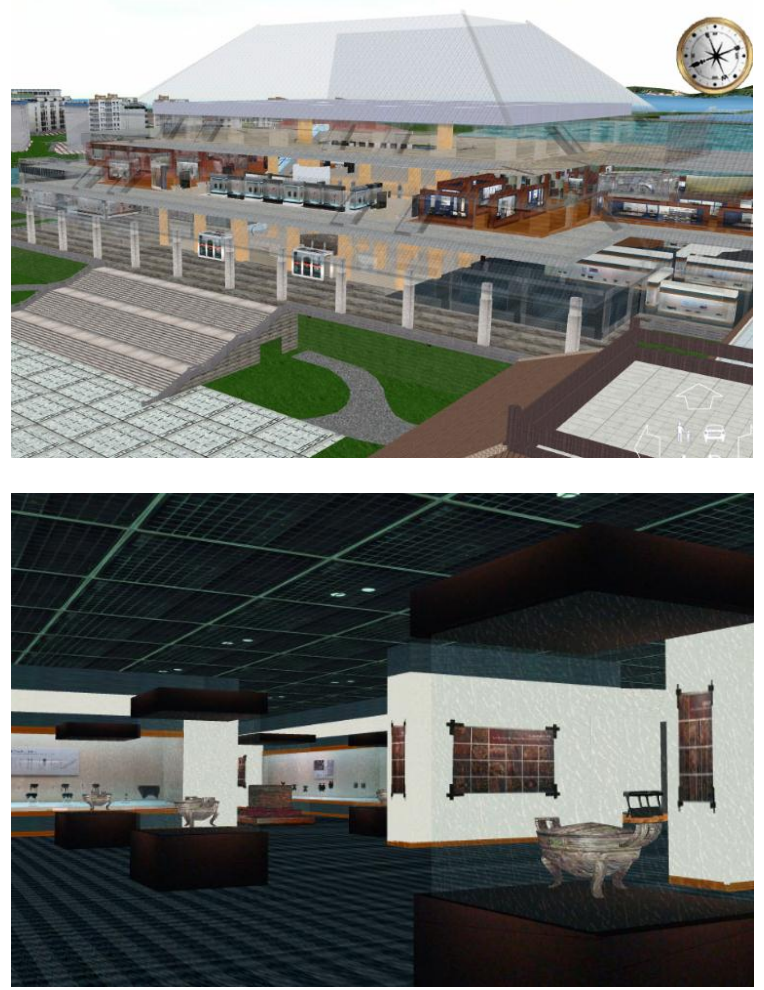

Figure 1.3D model of the museum

Besides the technical system requirements, user needs and wishes have to be investigated as different users will be using the application. Therefore users' requirements study was performed. More than 130 questionnaires were completed by visitors of the museum and a large group of employees of the museum were interviewed. Results of the study have shown that more than 50 percent of respondents consider 3D visualisation important and Bird's eye view of $3 \mathrm{D}$ model is preferred navigation environment. Several more preferences were specified as follows. The system should allow users to find their destinations dynamically which means the path should be computed based on user's current location and the path can be updated when user changes the destination. The system should be context-aware. It should provide information and services with respect to the type of the visitor. Two types of information are required: visit and services (Lin, 2007). Visit information relates to the exhibition such as theme, content, time and display location of exhibit. Visit information provides all relevant details for the main exhibitions. Service information includes the services provided in the museum for the convenience of the visitors during their visit such as checkrooms, dressing rooms and gift shops.

\section{3D MODEL}

The analysis in the former section shows that the $3 \mathrm{D}$ model is needed in this case for three main tasks: to represent the navigation environment, to support path finding and to provide context information about exhibitions and services of the museum. In order to achieve these goals, a hybrid 3D model including geometry, topology and semantics of the museum building is created: Firstly, a geometrical model with semantics is built to represent the navigation environment and provide context information. Secondly, a network which contains topology of the building is extracted from the geometrical model for path finding. Finally, a connection is created between the geometrical model and the network.

The geometrical model uses boundary representation (Figure 2). It subdivides the geometries of the museum into spaces and objects where the spaces represent corridors and exhibition rooms, and the objects represent the components of indoor space such as walls, ceilings, doors and exhibits. The semantics of the building are stored based on the geometrical model as an attribute of the object.

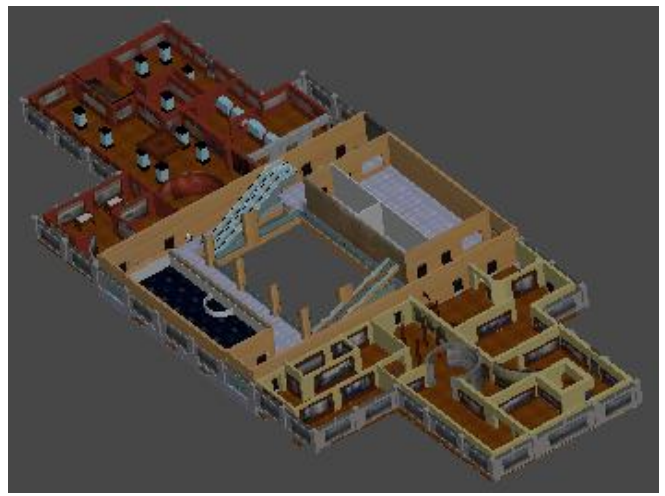

Figure 2. Geometric model of the building

The network contains two elements: nodes and edges. Nodes represent spatial components (spaces and objects) while edges stand for the connections between different components. Moving from one node to the other is allowed only when there is a link between them (Thill et al., 2011). The link indicates the distance between nodes in the extracted network. Due to the direct generation of $3 \mathrm{D}$ network is very challenging, the $2 \mathrm{D}$ network of each floor is firstly extracted and the topological relation between different floors is constructed by connecting special units (stairs and escalators) of adjacent floors represented as nodes. For each floor, the Constrained Delaunay Triangulation is used to subdivide the spaces, and the centres of triangles are taken as the nodes. Thus a space is represented by multiple nodes which are connected adjacently (Figure 3). Objects are indicated by one node which is connected to the nearest space node. The network is linked to geometrical model via IDs of nodes which are an attribute of the object.

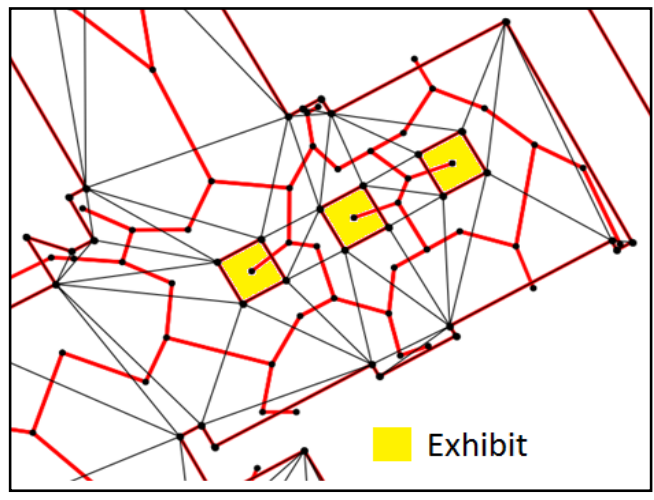

Figure 3. Extracted network 


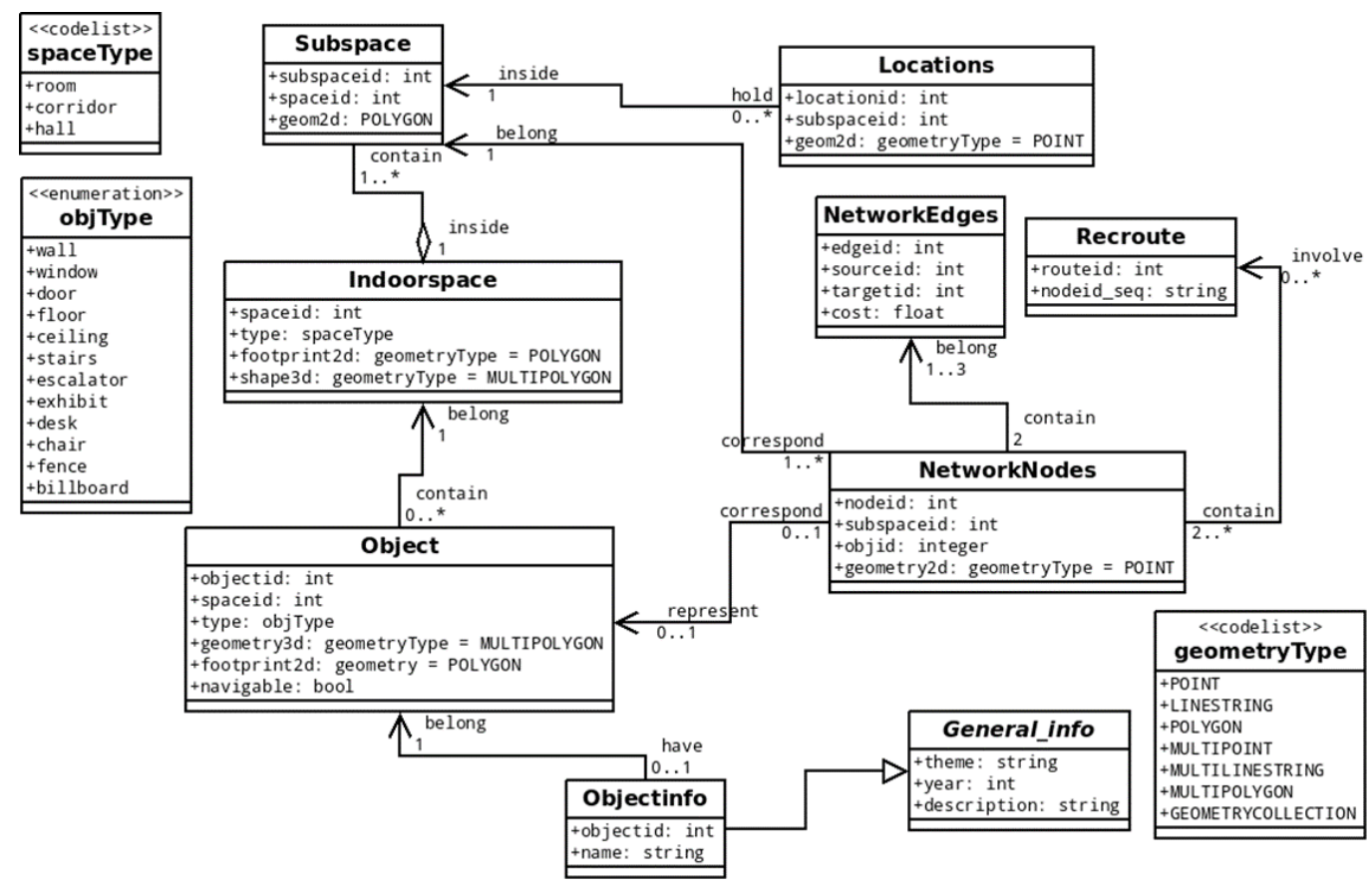

Figure 4. Data model

\section{IMPLEMENTATION}

In order to store all the information of the 3D model (geometry, topology and semantics) in one database, a new data model is designed. Finally a three layer system with application, web service and database is developed to provide localization, navigation and visualization services. A Graphic User Interface (GUI) of system is established based on Unity3D game engine to provide intuitive guidance and interaction for the visitors of Hubei Provincial Museum on a 3D model.

\subsection{Database}

The PostgreSQL database with PostGIS extension is chosen as it supports spatial objects and is a freeware database. A new data model is developed to store all information needed in one database and establish efficient connection between them (Figure 4). The model stores the geometric data and topologic network separately since this can decrease the time costs of processing spatial queries although it takes more space than the topology-enabled geometric model (Lee and Lee, 2010).

The model includes nine data classes for three main types of information. "Indoorspace", "Subspace" and "Object" are classes for geometric data. The "Indoorspace" and "Object" describes spaces and objects that were introduced in the previous section. The "Subspace" is defined as subdivisions of a space which is used to represent the probable area of the user's location. The floor plans of museum can be extracted automatically from the "Object" data. However, there are invalid geometries of the generated floor plan which need to be corrected manually. It is possible to retrieve the relevant geometries to represent the surrounding environment of the visitors instead of using the whole model which is too large to be visualized on a mobile device. The network is derived from the floor plan and used for routing "NetworkNodes" and "NetworkEdges" are data classes for nodes and edges of the network. "General_Info" and "ObjecInfo" are classes for context information of museum and exhibitions. The "Locations" class is created on the fly to store the user's location.

However, the geometric data stored in the database is not used for visualization by the application at the moment as issues to store and export the texture information of the model in database were encountered. To make the navigation environment realistic, a file-based 3D model with texture (COLLADA file) was used instead.

\subsection{System Architecture}

Different components of the system and interaction of these components are defined in system architecture. The conceptual model of the system architecture is depicted in Figure 5 where two types of components are distinguished: functional and system components. Functional components perform specific functions while system components support functional components to carry out these tasks.

The three functional components are: localization, navigation and 3D model visualization.

Localization component calculates the location of the mobile device carried by the user in the Hubei Provincial Museum. The output of this component is used as an input data for the navigation component.

Navigation component contains two functions: network extraction and path computation. The navigation network is semi-automatically extracted from the floor plan of the building in off-line phase. To compute the path, it retrieves the user's location from the localization component and the destination target from the application. Then the $\mathrm{A}^{*}$ algorithm provided by pgRouting extension of PostGIS is used to get the shortest path between the start and target points. 
$3 D$ model visualization component is responsible for visualization of the $3 \mathrm{D}$ model of the museum and calculated route on a mobile display.

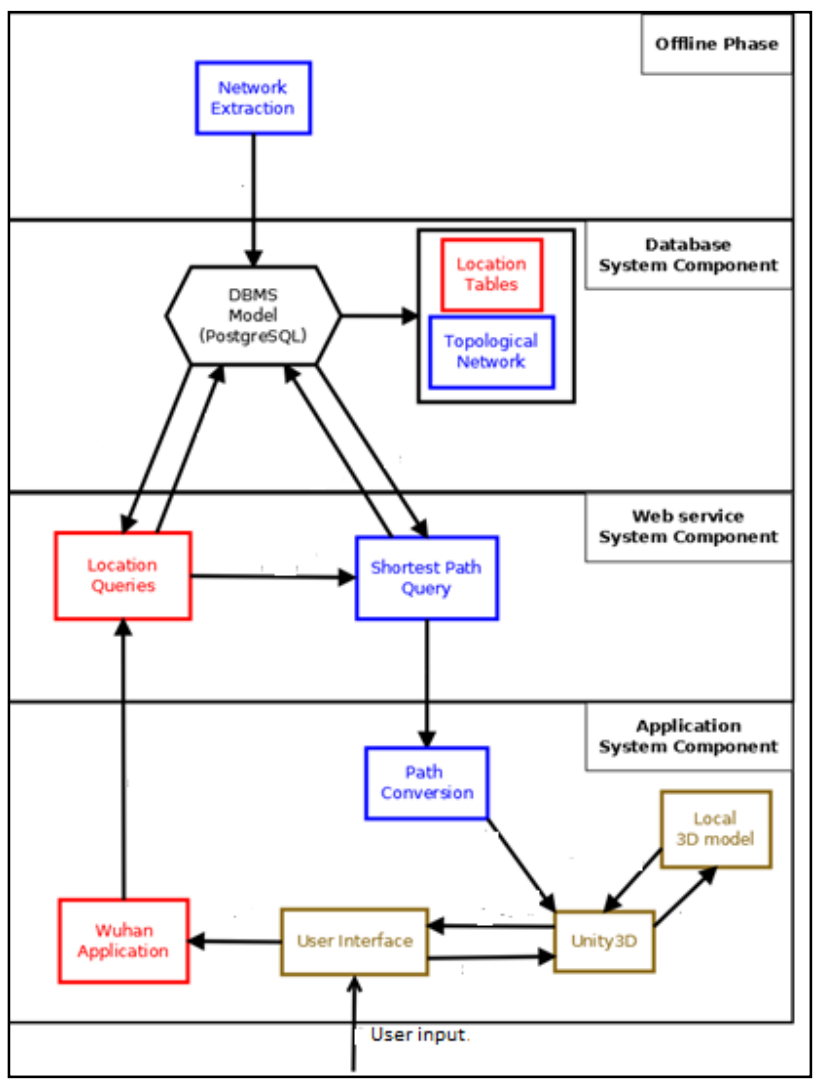

Figure 5. Conceptual model of the system

In order to support the introduced functions, the system is developed in three layers. The top layer is an Android application which allows users to find locations/objects of interest and view the path from their position to the target in a 3D model of museum. The bottom layer is the database where most data needed for the application is stored and path calculation is carried out. In addition, the web service functions as the middle layer between Android application and database by sending the request from application to the database and returning the query results.

\subsection{Graphic User Interface (GUI)}

The GUI of the application is developed using graphic layout tool of Android application and game engine Unity3D. The GUI provides guidance in the museum. Through the main menu the user can go to "Popular Exhibitions" where a list of most visited exhibitions is shown, "Recommended Routes" presents several recommended visiting routes or "Find Location" offers a search for the places that the user may want to go such as exhibitions, restroom and exit. All these three selections bring the 3D map which is the core component of the GUI to the user (Figure 6).

In the map view, the 3D Bird's eye view of the museum and the path defined by the user is presented. In order to visualize the 3D model on a mobile device, the Unity3D game engine is used. The 3D scene needs to be first created using Unity3D software, then a C\# script is written as a component of the scene to read the coordinates of the path and render it on the model. Finally the unity3D project needs to be exported to the main Android project as a library.
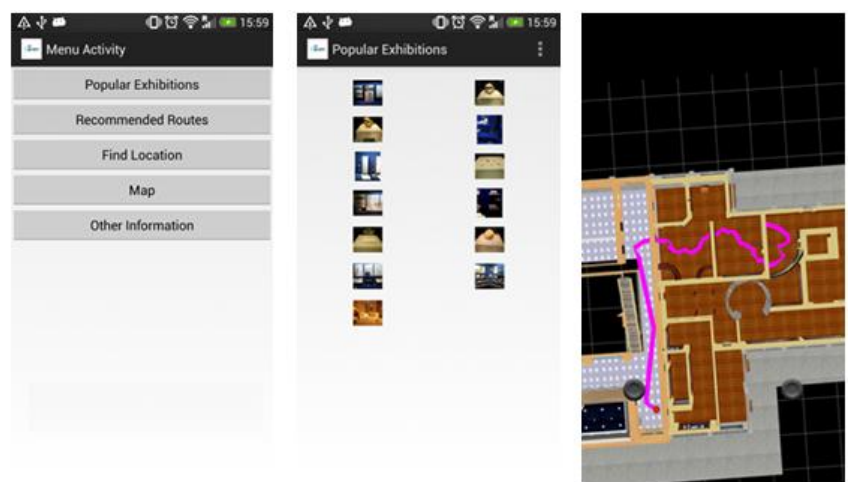

Figure 6. Left: main menu. Centre: popular exhibitions. Right: map view

More details about all components of the developed application can be found in the final report of the project on the website of MSc Geomatics programme at Delft University of Technology (http://www.tudelft.nl/studeren/masteropl/masteropleidingen/ge omatics/).

\section{CONCLUSIONS AND RECOMMENDATIONS}

This research project has clearly demonstrated that the proposed 3D model is valuable for indoor navigation since it can describe the location in object-level, provide context information of spatial objects (e.g., the museum and exhibitions), and more importantly offer a very realistic navigation environment as well as a good overview of it. These characteristics are exactly what users expect for an indoor navigation system. The developed application has many advantages compared to existing applications as Google maps, Wifarer or BuildNGO. The system architecture is able to ensure the high performance and security of data. All data that are necessary to make the application functional are stored in the database on an external server and most calculations are processed on a web server. This makes the client very lightweight and able to be installed as the user enters the museum. The network extraction method is smart and renewable. The whole process of the extraction is carried out automatically except a little manual work on cleaning the invalid geometries of floor plans. The network enables easy updates which are required since exhibitions might change during the time. The 3D model of the Hubei Provincial Museum is visualised on a mobile device using Unity3D game engine which ensures high level of performance, enables texture representation of spatial objects and provides interaction with the 3D model. The above mentioned characteristics ensure that the developed application can be easily adapted to any other building.

However there are still several aspects of the application could be improved in the future. The geometric data stored in database should be exported and sent to a mobile device dynamically representing the navigation environment. The context information of 3D model should be enriched and visualized on the screen of a mobile device. The automatically derived network needs further improvements to allow movement along a straight line, especially when certain exhibit items are not going to be considered. 


\section{ACKNOWLEDGEMENTS}

This project was completed under the umbrella of Joint Research Centre between TU Delft and Wuhan University and with the financial support of Delft Infrastructure and Mobility Initiative, Bureau BrowserBeest and Kadaster. The authors are thankful to all professors and students of Wuhan University who contributed to the successful completion of this project.

\section{REFERENCES}

Afyouni, I., Ray, C. and Claramunt, C., 2012. Spatial models for context-aware indoor navigation systems: A survey. Journal of Spatial Information Science, 4, pp. 85-123.

Android Apps on Google Play. BuildNGO - Indoor Navi, n.d. Web. 17 Oct. 2013. <https://play.google.com/store/apps/details?id=com.sails.buil dngo>.

Becker, T., Nagel, C., Kolbe, T. H., 2009, A Multilayered Space-Event Model for Navigation in Indoor Spaces. 3D Geo-Information Sciences, Lecture Notes in Geoinformation and Cartography, 2009, Part II, pp. 61-77.

Fallah, N., Apostolopoulos, I., Bekris, K., and Folmer, E., 2013. Indoor Human Navigation Systems: A Survey. Interacting with Computers, 25 (1), pp. 21-33

Google, Google Mobile, n.d. Web. 17 Oct. 2013. <http://www.google.c om/mobile/maps/>.

Le, M.H.V., Saragas, D., Webb, N., 2009. Indoor Navigation System for Handheld Devices. Worcester Polytechnic Institute, Worchester, Massachusetts, USA

Lee, J., 2004. A spatial access-oriented implementation of a 3-D GIS topological data model for urban entities. Geoinformatica, 8 (3), pp. 237-264

Lee, S., Lee, Y.J., 2010. Efficient topological data models for spatial qu eries in 3D GIS, in: A special joint symposium of ISPRSTechnical Com mission IV \& AutoCartoin conjunction with ASPRS/CaGIS 2010 Fall Sp ecialty Conference. Available at: http://www.isprs.org/proceedings/XX XVIII/part4/files/Lee Seokho.pdf, (Accessed 25 October 2013).

Lin, F.S., 2007. The study of domestic and foreign visitors' information needs in the national palace museum in Taiwan, International Association of Societies of Design Research, pp. 12-15

Thill, J. C., Dao, T. H. D., \& Zhou, Y., 2011. Traveling in the threedimensional city: applications in route planning, accessibility assessment, location analysis and beyond. Journal of Transport Geography, 19(3), pp. 405-421.

Wifarer Indoor Positioning, Indoor Positioning Technologies, n.d. Web. 17 Oct. 2013. 